\title{
Ozone clouds over the Atlantic
}

Paul Crutzen and Mark Lawrence

$\mathrm{O}$ zone in the atmosphere has many functions. Especially important to us is that it acts as a filter against solar ultraviolet radiation, protecting the biosphere from a large fraction of the biologically active and potentially harmful ultraviolet radiation of wavelengths less than about $325 \mathrm{~nm}$. About $90 \%$ of all ozone is located in the stratosphere (above 10 to $20 \mathrm{~km}$ ) and only 10\% in the troposphere (the region down to ground level). The two regions are separated, dynamically, by the tropopause, but, as reported by Suhre et al. on page 661 of this issue ${ }^{1}$, new measurements taken on airliners show that, contrary to earlier information, pockets of extremely high ozone concentration are somehow getting from the stratosphere into the troposphere over the tropical Atlantic.

Both regions are substantially affected by human activities. Ozone concentrations in the troposphere have increased, unlike those in the stratosphere. This is obvious during local episodes of photochemical smog near the ground, but also occurs in wider regions that are affected by anthropogenic emissions of hydrocarbons, carbon monoxide (CO) and nitric oxide (NO), such as the mid-latitude industrial zone of the Northern Hemisphere and the continental tropics and subtropics, which are strongly polluted by biomass burning during the dry season (Fig. 1). In high concentrations ozone can be deleterious to the biosphere, affecting human health, plant growth and agricultural productivity (ref. 2 and refs therein).

The effects of ozone and ultraviolet radiation in the troposphere are, however, not only negative. They produce hydroxyl $(\mathrm{OH})$ radicals, which are involved in the removal of almost all atmospheric gases emitted by human and natural activities - despite very low tropospheric concentrations, about 4 in $10^{14}$ molecules of air, hydroxyl's high reactivity makes it the detergent of the atmosphere. Most hydroxyl radicals are formed when ozone absorbs solar ultraviolet (UV-B) radiation of wavelengths less than $325 \mathrm{~nm}$. This leads to the production of electronically excited oxygen atoms which have enough energy to react with water vapour to produce hydroxyl radicals.

There is less total overhead ozone in the tropics than anywhere else, so more UV-B radiation penetrates. There is also more water in the tropical atmosphere, so the production of hydroxyl radicals is higher there than elsewhere. A quantitative understanding of the chemistry of the global atmosphere requires, therefore, good knowledge of the chemistry of the tropics and subtropics. This is all the more important as the greatest impact of human activities on atmospheric chemistry is probably going to occur in these parts of the world.

Unfortunately, information about ozone and other important chemical species is very much lacking in these regions. Regular vertical profiles of ozone are measured at only a handful of tropical stations. Past measurement campaigns have shown a high variability in space and time in the tropics and subtropics. For example, high concentrations are measured over large parts of the rural areas of South America, Africa and Southeast Asia during the dry season, when biomass burning releases a chemical mixture that is the precursor of photochemical smog (CO, hydrocarbons and $\mathrm{NO}_{x}$ ). Between 2 and $5 \times 10^{12} \mathrm{~kg}$ of biomass carbon are burned each year in the tropical and subtropical world ${ }^{3}$. This is dirty burning without emission controls. In comparison, global fossil fuel burning is about $5.5 \times 10^{12} \mathrm{~kg}$ a year.

In contrast, extremely low ozone concentrations have been measured in the cleanest region of our globe, that is, over the tropical Pacific. Vertical ozone soundings carried out from a ship between the Solomon Islands and Christmas Island ${ }^{4}$ showed verylow ozone volume mixing ratios of less than 10 p.p.b. (parts per billion) within a few kilometres of the sea surface. That was expected, owing to strong ozone loss by hydroxyl-forming reactions. Unlike measurements made anywhere else in the world, however, there was sometimes almost zero ozone concentrations in the upper troposphere. These low-ozone events appear to be related to intense convective activity and humidification of the air by evaporation of ice particles lifted up by the convection. Higher ozone values were generally

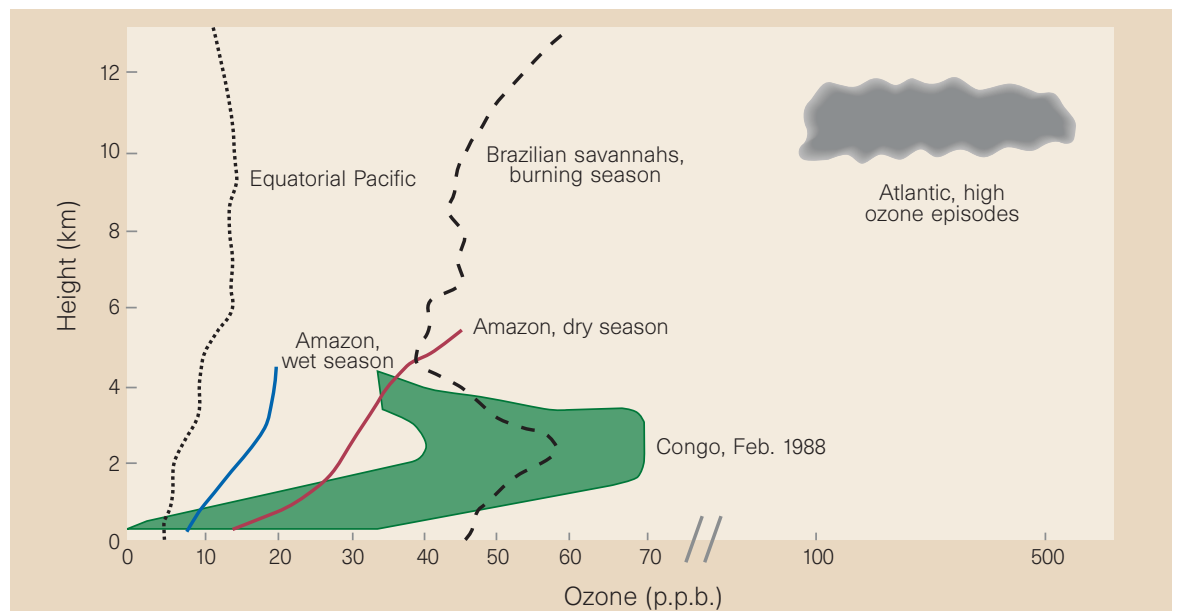

Figure 1 Ozone profiles in the tropics show the contrasts between dry and wet seasons, and between continental and marine sites. Episodes of extremely high ozone concentration, now seen over the Atlantic, are unlike anything reported before. These air pockets must somehow be getting into the troposphere from the stratosphere above. found in drier air in the upper troposphere, between 10 and $12 \mathrm{~km}$, indicating some downward transfer of ozone from the dry stratosphere. But $\mathrm{O}_{3}$ ratios remained below 30 p.p.b.

Now for the next great surprise in the ozone saga: over the tropical Atlantic, between 10 and $12 \mathrm{~km}$, Suhre et al. ${ }^{1}$ found extremely high ozone concentrations of up to 500 p.p.b., together with high humidities, in convective regions $5-80 \mathrm{~km}$ across. These observations were made by automated instrumentation on an ordinary passenger aircraft. How can this be explained?

Leaving aside the unlikely explanation that lightning causes these very high ozone peaks, they must be due to transport from the stratosphere. There are two possibilities: downward turbulent transfer through the tropopause, caused by convection; or advection of air masses from outside the tropics through a break in the tropopause that is known to exist in the subtropics. Contrary to the Pacific measurements, the high ozone values were not accompanied by low water concentrations (which is a characteristic of stratospheric air). So the air must have been humidified, possibly through the evaporation of ice particles falling from the anvils of thunderclouds, accompanied by downward mixing of the ozone-rich air parcels.

Why this happens over the Atlantic, yet was not observed over the convective part of the tropical Pacific, requires further exploration. Unfortunately, the chemical instrumentation on the MOZAIC aircraft is restricted to $\mathrm{O}_{3}$ and $\mathrm{H}_{2} \mathrm{O}$. It would have been useful to have measurements of other chemical constituents, such as $\mathrm{CO}, \mathrm{CO}_{2}, \mathrm{~N}_{2} \mathrm{O}$ and halocarbons: if the high-ozone pockets come from the extra-tropical stratosphere, the air would have been chemically processed during its journey, leading to lower amounts of $\mathrm{N}_{2} \mathrm{O}$ and halocarbons, and higher amounts of more reactive nitrogen and halogen oxides - with potentially interesting chemical behaviour in the presence of ice. 
The impact of the high ozone intrusions on the overall photochemistry of the tropical troposphere cannot be estimated from the data of Suhre et al. The authors have, however, clearly demonstrated the great opportunities and cost effectiveness offered by instrumentation on commercial aircraft. The results show again very clearly how little we know about tropospheric ozone in the tropics and subtropics, which are so important photochemically. Until these regions are much more intensely researched, the quantitative basis for chemical studies will remain weak. The participation of scientists from the tropics and subtropics in this research should be encouraged.

Paul Crutzen and Mark Lawrence are in the MaxPlanck-Institut für Chemie, Abteilung Chemie der Atmosphäre, Postfach 3060, 55020 Mainz, Germany.

1. Suhre, K. et al. Nature 388, 661-663 (1997).

2. Chameides, W. L., Kasibhatla, P. S., Yienger, J. \& Levy, H. Science 264, 74-77 (1994).

3. Crutzen, P. J. \& Andreae, M. O. Science 250, 1669-1678 (1990).

\section{Fusion induces tumour rejection}

\section{Ian Hart and Camilo Colaco}

$\mathrm{T}$ he war on cancer has been waged with ever-increasing intensity over the past 30 years or so, using the weapons of surgery, radiotherapy and chemotherapy. But it has resulted, to date, in only limited advances. This has led to calls for the main directions of research to be realigned, from treatment to prevention ${ }^{1}$. But cancer therapy will be given a renewed impetus by a paper published in Nature Medicine by Kufe and co-workers', who describe an elegant but simple strategy for the treatment of cancer using immunotherapy.

The idea of immunotherapy as a new approach may seem to be a contradiction in terms, since strategies based on this concept have been applied clinically for over 100 years, with limited success. Nonetheless, there is renewed optimism ${ }^{3,4}$, largely based on a better understanding of how our cytotoxic $\mathrm{T}$ lymphocytes (CTLs) respond to tumour cells ${ }^{5}$ (Fig. 1). Central to the induction of CTL responses are professional antigen-presenting cells. These include the B lymphocytes, mononuclear phagocytes and dendritic cells, which present antigen to the immune system in the context of major histocompatibility complex (MHC) class I and class II molecules ${ }^{6}$.

In a host of experiments over the past few years, dendritic cells have been used to process and present tumour antigens. This has generally been achieved by pulsing cultured dendritic cells with peptides eluted from class I MHC molecules ${ }^{7}$, tumour cell membranes ${ }^{7}$ or RNA derived from the neoplastic cells ${ }^{8}$. Kufe and co-workers have pursued the alternative strategy of introducing tumour antigens into dendritic cells in the most straightforward way - by fusing entire tumour cells with cultured dendritic cells.

The authors found that when cells from the murine MC38 carcinoma were fused with bone-marrow-derived dendritic cells, they obtained fusion hybrids. These were then used to immunize syngeneic (genetically identical) mice, and they generated a strong antitumour activity in these animals. Interestingly, this antitumour activity seemed to involve both CD4- and CD8-positive T cells, in MHC class I- and II-restricted responses, respectively. This is in contrast to the largely CD8-positive, class I-restricted responses that have been observed when dendritic cells pulsed with antigen have been used as immunogens ${ }^{7}$. More excitingly and potentially of greater clinical significance - this antitumour activity occurred not only against primary tumours, but also against pre-established metastatic disease ${ }^{3}$.

The fusion of tumour cells with antigenpresenting cells is not a new concept, and it has already been achieved with activated B cells'. However, as Kufe and colleagues point out, the use of dendritic cells offers two additional advantages - they can prime naive CTLs, and they are easily accessible from peripheral blood or bone marrow. This availability of donor cells would be of considerable benefit in any human application. But what of the tumour-cell side? Obviously, the single 'swallow' of the MC38 tumour does not a 'summer make', and both the procedure and the magnitude of the antitumour effects will need to be verified in further animal models. Moreover, efficacy against rodent tumours is not a guarantee of success against human cancers. The authors warn of the inherent limitations of animal models but, with their approach, it may be the technical rather than conceptual aspects that limit easy transfer to the clinic.

Fusion followed by selection means that, if the procedure is to be applied to individual human tumours, the neoplastic cells must have the capacity for continuous in vitro proliferation - not necessarily an attribute of all primary cell cultures. In the work by Kufe and colleagues, no selectable markers seem to have been incorporated into the fusion partners. This, of course, is not a problem when pure populations of tumour cells are used. But if mixed populations are derived from patient material, how can we guarantee that anti-self responses will not be generated against normal cells? These might fuse with the dendritic cells as easily as the cancer cells do, and lead to undesirable anti-self responses on injection. One way to obviate such potential problems might be to create universal fuser lines of representative tumours, rather than relying on material from individual patients.

The work of Kufe and co-workers - with its dramatic effects on tumour burden and immunoprotection - is complementary to the encouraging results of other groups, which have used dendritic cells to stimulate naive $\mathrm{T}$ cells. For example, the pulsing of dendritic cells with tumour antigens in vitro generates both tumour-specific CTLs ${ }^{10}$ and tumour resistance in vivo ${ }^{8}$. The rejection of established tumour metastases ${ }^{3,7-10}$ parallels the anti-graft rejection responses in transplantation immunology induced by donor dendritic cells ${ }^{11}$. This potency of the immune response that is elicited by dendritic cells is also illustrated by their ability to break neonatal tolerance ${ }^{12}$.

These results are promising because they represent new approaches to targeted immunotherapy that are based on a greater appreciation of the mechanisms by which

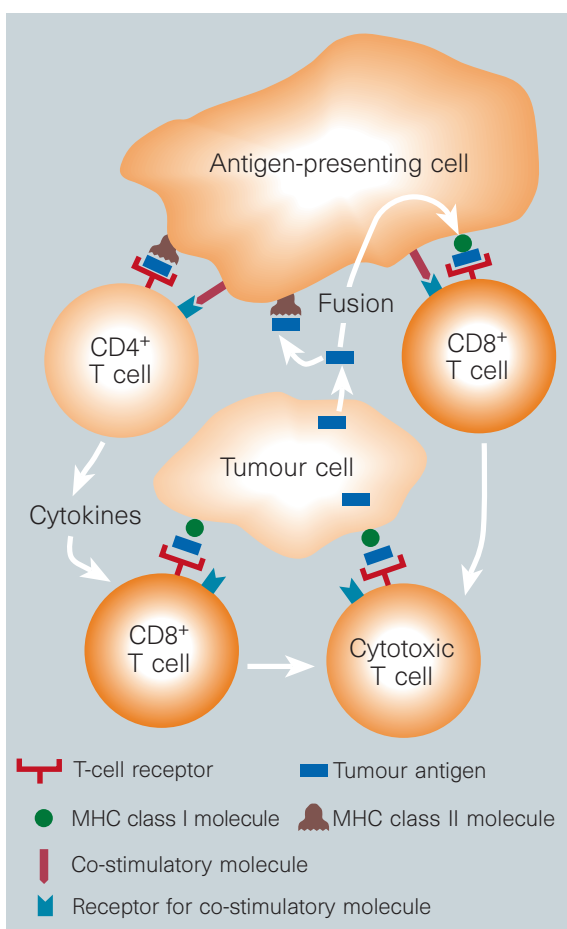

Figure 1 Kufe and colleagues ${ }^{2}$ have developed a new strategy to activate the response of cytotoxic T lymphocytes against tumour cells. Precytotoxic CD8-positive T lymphocytes may be primed directly by antigen-presenting cells, which present tumour antigens on a major histocompatibility complex (MHC) class I molecule. Presentation of tumour antigen by MHC class II molecules to CD4-positive helper T lymphocytes leads to the release of stimulatory cytokines, which activate the pre-cytotoxic CD8positive $\mathrm{T}$ lymphocytes. By fusing one group of antigen-presenting cells - the dendritic cells with entire tumour cells, the tumour antigens can be expressed and used to directly activate naive $\mathrm{T}$ lymphocytes. 